(C)2009 IEEE. Personal use of this material is permitted. However, permission to reprint/republish this material for advertising or promotional purposes or for creating new collective works for resale or redistribution to servers or lists, or to reuse any copyrighted component of this work in other works must be obtained from the IEEE. 


\title{
A Fuzzy Inference Model for Risk based Informed Decision-Making in e-Business
}

\author{
Omar Hussain, Tharam Dillon, Elizabeth Chang and Farookh Hussain \\ Digital Ecosystems and Business Intelligence Institute, Curtin University of Technology, Australia \\ e-mail: o.hussain@cbs.curtin.edu.au
}

\begin{abstract}
Since its invention, the web has considerably advanced and it now provides its users with sophisticated technologies and processes for facilitating interactions. This has enabled users to complete their tasks by efficient ways and in turn increase their productivity. But in such an environment, it is imperative for a user to make informed decisions in a proactive way by which it can achieve its aims and maximize its interaction experience. To achieve that, various approaches have been proposed by which a user can make an interaction-based decision in web-based ecommerce. But at the same time the notion of risk has been considerably ignored in that domain; even though it has been mentioned in the literature that risk plays an important part while decisionmaking in any domain. So in order to have such an analysis of risk while decision-making, in this paper we highlight the importance of risk and then propose a fuzzy inference model for assisting risk-based decision making in e-commerce business interactions.
\end{abstract}

Keywords- Perceived risk, Risk propensity, Decision making.

\section{INTRODUCTION}

The rapid pace of development of the internet for facilitating e-commerce interactions has provided users with sophisticated technologies which eases the process of carrying out their activities and the ability to complete their tasks in less time. This has reduced the delays associated with the conventional method of interactions thus boosting user's efficiency as well as helping them to improve their sales and productivity. But as mentioned by Chang et al. [1] 'The dynamic, open and convenient web environment not only boosts business potential and the economy but also creates concerns of security, trust, privacy and risks'. So the users before utilizing the provided facilities to their advantage, should consider and analyse beforehand these factors in order to make sure that they achieve what they desire, or get the maximum output in their interactions. In the literature, various approaches have been proposed for the interaction initiating agent to make an informed interactionbased decision in e-business interactions [2-5]. But from an analysis of those approaches it can be inferred that the notion of perceived risk has been considerably ignored and has not been considered to determine its impact while making an informed interaction-based decision. Those approaches by not considering the risk in an interaction while decisionmaking ignore and do not take into consideration all the concepts that are required to be considered while decision making. Risk plays a central role in deciding whether to proceed with an interaction or not. It can broadly be defined as an attribute of decision-making that reflects the variance of its different possible outcomes. We term the category of risk that we are speaking about in this case as the 'transactional risk'. Transactional risk represents the level of risk associated with the successful completion of the business activity. This is different from the other risks associated with the other aspects of business activity such as security risks, privacy risks etc. Further in the paper we term the transactional risk as the perceived risk in the business interaction. In the domain of e-business, the analysis of perceived risk will give the interaction initiating agent the level of failure that it can experience in achieving its desired outcomes; as well the possible financial loss in its invested resources. This type of output is not represented by the analysis of factors like trust, security or privacy; and thus risk commands a central role in any discussion that is related to an interaction. Due to space limitations we cannot do a detailed comparison of risk with other concepts like trust and security.

In this paper, we propose an approach which addresses this issue and by which the interaction initiating agent can consider the quantified perceived risk in forming an interaction and then utilize it to decide on in forming an interaction with the other agent. The paper is organized in the following sections. In section 2 we highlight the importance of considering the risk propensity of the interaction initiating agent while decision-making. In section 3 we develop a fuzzy inference system for decision-making and define the rules. In section 4 we explain the proposed approach by using an example and discuss the results in detail. Finally in section 5 we conclude the paper.

\section{RISK PROPENSITY WhILE DECISION-MAKING}

In order to consider the level of risk while decisionmaking, it is important to first assess it according to its object of analysis in e-commerce business. While quantifying perceived risk in the domain of e-business environments, the likelihood and magnitude of perceived risk should be determined, as the need to determine these characteristics of risk in such type of interactions is important. So in order to consider these characteristics, we define perceived risk in ecommerce business as a combination of its two subcategories namely performance risk and financial risk. We define the two interacting agents as the risk assessing agent and the risk assessed agent. The risk assessing agent is termed as the initiator of the interaction and the risk assessed agent is the agent with whom it interacts with. Performance risk is defined as the likelihood of the risk assessed agent in not providing the risk assessing agent with what it desires or expects in the time period of its interaction, resulting in the negative outcome or failure in the interaction [6]. The expectations of the interaction are formed beforehand after a 
series of negotiations between the interacting agents. Financial risk is defined as the degree and magnitude of loss to the risk assessing agent in the interaction due to the performance risk of the risk assessed agent [7]. In our recent work, we have proposed a methodology by which the risk assessing agent determines the numeric [8] and linguistic level [9] of risk in an interaction as a combination of these subcategories.

Once this is done then the risk assessing agent has to utilize the determined level of perceived risk to decide on its future course of interaction with the other agent. Alternatively, it may be the case that the risk assessing agent may have to make a decision about choosing an agent to interact with from a set of agents, after managing the level of perceived risk in interacting with each of them. To assist itself during this process; the risk assessing agent should analyze the determined level of perceived risk in interacting with an agent, according to its risk propensity in the interaction. In this paper, we propose such a methodology by which the risk assessing agent can utilize the determined level of perceived risk to decide whether to form an interaction with a risk assessed agent. Since the decisionmaking process is best understood when it is expressed semantically, we utilize a fuzzy inference system to propose the methodology for assisting the risk assessing agent in decision-making. In the next sub-section, we define the term 'risk propensity' and how it impacts upon the risk assessing agent while making an interaction-based decision.

\section{A. Risk Propensity and its effect on Decision Making}

While decision making, 'Risk Propensity' defines the risk assessing agent's risk taking nature or its risk attitude in the interaction. It also defines its current tendency towards taking the level of risks in an interaction. So in an interaction, the risk propensity of the risk assessing agent is the main factor in determining how it will behave or react to the level of perceived risk and what its interaction based decision output is going to be [10]. It is important to note that no two risk assessing agents might have the same risk attitude, and with the variation in their risk attitudes their approach to decision making in the interaction varies. Also, the risk attitude of an agent might not be the same throughout. Subsequently while making the decision, it is very important for the risk assessing agent to first ascertain its accurate risk propensity at that given period of time and then to determine its impact on the level of perceived risk in the interaction, for making an informed determination on an interaction.

We propose that the risk assessing agent while determining semantically the risk-based decision output, should consider the impact of its risk propensity on the determined linguistic level of perceived risk in interacting with the risk assessed agent, which is determined by our previous work in Hussain et al. [9]. This is because, the linguistic level of perceived risk has semantics associated with it, which can be utilized to better ascertain the semantic decision output in the interaction. To determine the linguistic level of perceived risk in an interaction, we utilized a fuzzy inference system in [9] that has 6 fuzzy sets or predicates.
The different fuzzy sets are: Extremely Low (EL), Low (L), Low Medium (LM), Medium High (MH), High $(\mathrm{H})$ and Extremely High (EH). The risk propensity of the risk assessing agent should be determined from a spectrum or range which specifies all the different possible risk taking natures or risk attitudes possible in an interaction. We define the spectrum of the risk propensity of the risk assessing agent as having three broad categories. They are:

- Risk Averse (RA): 'Risk Averse' is defined as that attitude of the risk assessing agent where it wants to take only minimal risk in the interaction. We consider that the agents with a risk propensity level of 'risk averse' accept the magnitude of perceived risk up to $E L=1$ in interacting with a risk assessed agent.

- Risk Neutral (RN): 'Risk Neutral' is defined as that attitude of the risk assessing agent where it wants to proceed in the interaction only if the advantages that it will achieve will outweigh the costs involved. In other words, we consider that agents with a risk propensity of risk neutral do not totally avoid risk as done by those agents of a risk averse nature, and will accept the perceived risk in the interaction up to the level of $\mathrm{LM}=1$.

- Risk Taking (RT): 'Risk Taking' is defined as that attitude of the risk assessing agent where it is indifferent to any level of perceived risk and is ready to interact with a risk assessing agent, no matter what level of perceived risk is present in the interaction. We consider that the agents with a risk propensity nature of risk taking, will accept the magnitude of perceived risk up to $\mathrm{EH}=1$ in interacting with a risk assessed agent.

The risk attitudes of the risk assessing agent in terms of accepting the magnitude of perceived risk in the interaction can be arranged in the order of $\mathrm{RA}<\mathrm{RN}<\mathrm{RT}$. In order to determine on an interaction, the risk assessing agent has to first choose its risk propensity level or risk attitude from the spectrum. It is possible that the risk propensity level of the risk assessing agent might not always be a crisp value which corresponds totally to a given level, but might overlap across the different possible levels. To take such scenarios into account, we propose using a fuzzy inference system to capture the fuzziness of the risk attitude of the risk assessing agent, and then utilize it to accurately determine its impact on the level of perceived risk in the interaction. In the following sections, we define and develop a fuzzy inference model which quantifies the risk propensity of the risk assessing agent and then determines its impact on the level of perceived risk in interacting with the interacting agent, in order to recommend an interaction-based decision to the risk assessing agent.

\section{B. Defining the Fuzzy sets and the Membership function for the Input: Risk Propensity (RP) of the risk assessing agent}

The input to the system 'Risk Propensity' (RP) of the risk assessing agent represents its quantified risk attitude or its risk taking nature in the interaction. In the previous subsection, the spectrum over which the possible risk attitude of the risk assessing agent extends is defined. In order to consider the levels from that spectrum in our fuzzy inference 
model, we define the universe of discourse (UoD) of the input variable to the system 'Risk Propensity' of the risk assessing agent in the range of $1-5 ;\{1,2,3,4,5\}$ where each element represents a numeric value and is unit-less. To classify different fuzzy sets for the input variable 'Risk Propensity' of the risk assessing agent we divide the universe of discourse into 3 predicates, 'Risk Averse', 'Risk Neutral' and 'Risk Taking'. The membership function for the input 'Risk Propensity' of the risk assessing agent is such that it is a combination of a triangle and straight lines as shown in Figure 1. The membership function is used to determine the strength to which a value from the $\mathrm{UoD}$ of the input variable quantifies to the defined predicates of the risk attitude of the risk assessing agent.

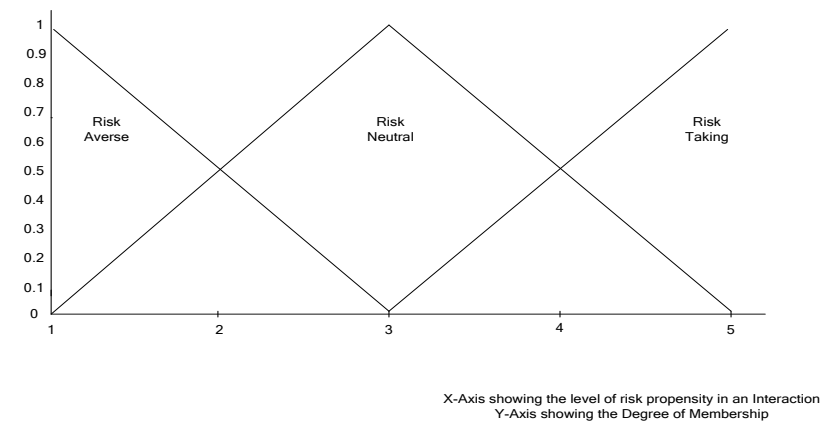

Fig. 1. Membership function for the input: Risk Propensity

\section{Defining the Fuzzy sets and the Membership function for the Input: Perceived Risk (PR) in the Interaction}

The input variable 'Perceived Risk (PR)' in the interaction represents the determined level and magnitude of perceived risk in interacting with a risk assessing agent, with whom the risk assessing agent has to determine on an interaction. In our previous work, we defined six fuzzy sets or predicates on which the level of perceived risk is determined. The membership function for the input 'Perceived Risk' is shown in Figure 2.

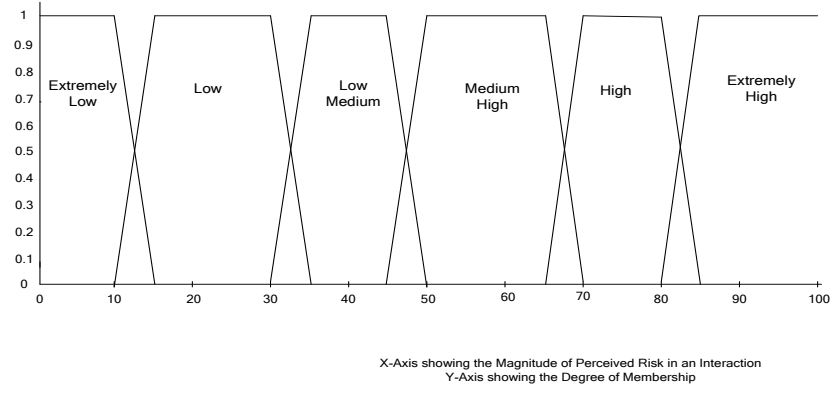

Fig. 2. Membership function for the input: Perceived Risk

\section{Defining the Fuzzy sets and the Membership function for the Output: Recommended Risk-based Decision (RRD)}

The fuzzy inference system computes an output specifying the Recommended Risk-based Decision (RRD), based on the inputs given to it. This recommended risk-based decision is the result of the effect of the risk propensity of the risk assessing agent on the perceived risk in interacting with the interacting agent. We consider a range of $0-10 ;\{0,1$, $2, \ldots, 10\}$ as the universe of discourse (UoD) while determining the recommended risk-based decision output by the system. As our aim is to develop a fuzzy inference system which assists the risk assessing agent in making an informed interaction-based decision with a risk assessed agent, the fuzzy sets for the output variable are defined such that there are two predicates in them. They are 'Proceed' $(\mathrm{P})$ and 'Don't Proceed' (DP) which represents the two possibilities for the risk assessing agent to consider while decision making in an interaction. The membership function for the output 'Recommended Risk-based Decision' in the interaction is defined as an intersection of straight lines spread over the universe of discourse for the fuzzy variable, as shown in Figure 3.

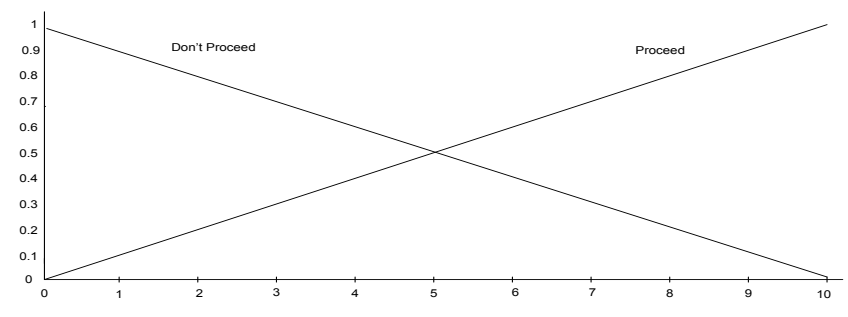

X-Axis showing the Recommended RIsk-based Decision in the interaction
Y-Axis showing the Degree of Membership

Fig. 3. Membership function for the output: Recommended Risk-based Decision

Once the input variables have been transformed into fuzzy sets by using their defined membership function, they must then be processed in the inference engine of the fuzzy system to determine the impact of the risk propensity of the risk assessing agent on the perceived risk in interacting with the risk assessed agent by using the defined rules. In the next section, we define the rules for the fuzzy inference system by which it recommends a decision output to the risk assessing agent in forming an interaction with a risk assessed agent.

\section{Defining The RULes For FuZZY ReCOMMENDED RISK-BASED DECISION MODEL}

With the change in the risk propensity levels the way how the risk assessing agent assesses the perceived risk in interacting with a risk assessed agent changes. Hence, it is important for the risk assessing agent to first ascertain its accurate risk propensity or its risk attitude and accordingly decide on the future course of the interaction. In the previous section, we defined the levels of acceptable perceived risk by the risk assessing agent only according to the crisp levels of its risk propensity. These levels of acceptable perceived risk in the interaction will change when the risk propensity of the risk assessing agent overlaps different levels. So, to take into consideration the intermediate values of the risk propensity, in Table 1 we define by using fuzzy rules the acceptable levels of perceived risk by the risk assessing agent, according to the various levels of its risk propensity. Furthermore, while defining the fuzzy rules, if the risk assessing agent's 
risk propensity is a combination of different attitudes, then we consider the 'Maximum Risk Attitude' (MRA) of the risk assessing agent and based on that determine the 'Maximum Acceptable Perceived Risk Level' (MARL) in the interaction. As mentioned in the last section, the nature of the risk taking attitude of the risk assessing agent in the interaction is in the order of: $\mathrm{RA}<\mathrm{RN}<\mathrm{RT}$. Hence, if a risk assessing agent's risk propensity nature is a combination of levels RA and RN with DOM 0.4 and 0.6 respectively, then the maximum risk attitude or maximum risk propensity nature of the risk assessing agent is $\mathrm{RN}=0.6$. Based on this level and by using fuzzy rules defined in Table 1, the risk assessing agent should determine the Maximum Acceptable Perceived Risk Level (MARL) in interacting with the risk assessed agent.

TABLE I. FuZZy RULES TO DETERMINE THE MARL OF THE RISK

\begin{tabular}{|l|l|l|l|}
\hline \multicolumn{5}{|c|}{ ASSESSING AGENT } \\
\hline If & $\mathrm{MRA}$ & & $\mathrm{MARL}$ \\
\hline If & $\mathrm{RA}=1$ & then & $\mathrm{EL}=1$ \\
\hline If & $\mathrm{RN}=0.1$ & then & $\mathrm{L}=0.2$ \\
\hline If & $\mathrm{RN}=0.3$ & then & $\mathrm{L}=0.4$ \\
\hline If & $\mathrm{RN}=0.4$ & then & $\mathrm{L}=0.6$ \\
\hline If & $\mathrm{RN}=0.5$ & then & $\mathrm{L}=0.8$ \\
\hline If & $\mathrm{RN}=0.6$ & then & $\mathrm{L}=1$ \\
\hline If & $\mathrm{RN}=0.7$ & then & $\mathrm{L}$ \\
\hline If & $\mathrm{RN}=0.8$ & then & $\mathrm{LM}=0.4$ \\
\hline If & $\mathrm{RN}=0.9$ & then & $\mathrm{LM}=0.6$ \\
\hline If & $\mathrm{RN}=1$ & then & $\mathrm{LM}=0.8$ \\
\hline If & $\mathrm{RT}=0.1$ & then & $\mathrm{LM}=1$ \\
\hline If & $\mathrm{RT}=0.2$ & then & $\mathrm{MH}=0.34$ \\
\hline If & $\mathrm{RT}=0.3$ & then & $\mathrm{MH}=1$ \\
\hline If & $\mathrm{RT}=0.4$ & then & $\mathrm{H}=0.34$ \\
\hline If & $\mathrm{RT}=0.5$ & then & $\mathrm{H}=0.67$ \\
\hline If & $\mathrm{RT}=0.6$ & then & $\mathrm{H}=1$ \\
\hline If & $\mathrm{RT}=0.7$ & then & $\mathrm{EH}=0.34$ \\
\hline If & $\mathrm{RT}=0.8$ & then & $\mathrm{EH}=0.67$ \\
\hline If & $\mathrm{RT}=0.9$ & then & $\mathrm{EH}=0.99$ \\
\hline If & $\mathrm{RT}=1$ & then & $\mathrm{EH}=1$ \\
\hline
\end{tabular}

It is highly possible that a risk assessing agent by utilizing the our proposed approach in Hussain et al. [9] determines that there is more than one fuzzy set or predicate of Perceived Risk present in its interaction with the risk assessed agent. In such scenarios three possibilities arise here according to the risk propensity nature of the risk assessing agent:

- the levels of perceived risk in the interaction are totally acceptable to the risk assessing agent;

- the levels of perceived risk in the interaction are totally unacceptable to the risk assessing agent;

- the level/s of perceived risk in the interaction is/are partially acceptable to the risk assessing agent.

In each case, the recommended output from the fuzzy inference system to the risk assessing agent varies according to the level of its acceptance of the perceived risk in the interaction, according to its risk attitude or risk propensity nature. So while decision-making, the risk assessing agent should consider each predicate of perceived risk in interacting with the risk assessed agent with the degree of magnitude of its occurrence and then determine the impact of its risk propensity on it.

In order for the fuzzy inference system to consider such cases, we introduce a variable called 'Possible to Proceed in the Interaction at this stage' (Poss) when defining the fuzzy rules for the recommended risk-based decision output. This variable is determined for each predicate of perceived risk and it represents whether or not that predicate along with its magnitude of occurrence is acceptable to the risk assessing agent according to its risk attitude or risk propensity, and depending on that, whether it should 'Proceed' or 'Don't Proceed' in the interaction. The output which the variable gives applies only to that predicate of perceived risk on which it is determined. If there are more than one level or predicate of perceived risk in the interaction, then the fuzzy system will consider each predicate of perceived risk and then determine the value for the variable 'Possible to Proceed in the Interaction at this stage' (Poss) for each of those predicates. We term the predicate of perceived risk which the risk assessing agent currently examines and determines the value of the variable 'Poss' as 'Current Risk Level' (CRL). The risk propensity of the risk assessing agent is represented by three fuzzy sets or predicates as shown in Figure 1, and the perceived risk in the interaction is represented by six fuzzy sets or predicates as shown in Figure 2. In Table 2 we define the rules for the system to make a risk-based decision in an interaction.

TABLE II. RULES For THE FUZZY RECOMMENDED RISK-BASED

\begin{tabular}{|l|l|l|l|l|l|l|l|l|l|}
\hline & CRL & & CRA & & RRD & & Poss & & RRD \\
\hline If & EL & and & RA & then & P & if & 1 & else & DP \\
\hline If & L & and & RA & then & P & if & 1 & else & DP \\
\hline If & LM & and & RA & then & P & if & 1 & else & DP \\
\hline If & MH & and & RA & then & P & if & 1 & else & DP \\
\hline If & H & and & RA & then & P & if & 1 & else & DP \\
\hline If & EH & and & RA & then & P & if & 1 & else & DP \\
\hline If & EL & and & RN & then & P & if & 1 & else & DP \\
\hline If & L & and & RN & then & P & if & 1 & else & DP \\
\hline If & LM & and & RN & then & P & if & 1 & else & DP \\
\hline If & $\mathrm{MH}$ & and & RN & then & P & if & 1 & else & DP \\
\hline If & $\mathrm{H}$ & and & RN & then & P & if & 1 & else & DP \\
\hline If & EH & and & RN & then & P & if & 1 & else & DP \\
\hline If & EL & and & RT & then & P & if & 1 & else & DP \\
\hline If & L & and & RT & then & P & if & 1 & else & DP \\
\hline If & LM & and & RT & then & P & if & 1 & else & DP \\
\hline If & MH & and & RT & then & P & if & 1 & else & DP \\
\hline If & $\mathrm{H}$ & and & RT & then & P & if & 1 & else & DP \\
\hline If & EH & and & RT & then & P & if & 1 & else & DP \\
\hline
\end{tabular}

'CRA' in Table 2 represents the fuzzy sets to which the risk propensity of the risk assessing agent quantifies to its defined membership function. The value for the variable 'Poss' in Table 2 for each of the 'CRL' being considered can be determined from Table 3, by taking into consideration the 'MRA' of the risk assessing agent and then determining if the 'CRL' is less than or equal to the 'MARL'. If this is the case, then the value of 'Poss' will be 1 for that 'CRL' in Table 2 otherwise it will be 0 .

Once the fuzzy rules of Table 2 have been evaluated, then they must be aggregated and defuzzified in order to obtain a crisp value on the output membership function. For 
TABLE III. FuZzy RULEs to Determine The VALUe For The VARIABLE 'POSS'

\begin{tabular}{|l|l|l|l|l|l|l|l|}
\hline & $\mathrm{MRA}$ & & $\mathrm{CRL} \leq$ & & Poss & & Poss \\
\hline If & $\mathrm{RA}=1$ & and & $\mathrm{EL}=1$ & then & 1 & else & 0 \\
\hline If & $\mathrm{RN}=0.1$ & and & $\mathrm{L}=0.2$ & then & 1 & else & 0 \\
\hline If & $\mathrm{RN}=0.2$ & and & $\mathrm{L}=0.4$ & then & 1 & else & 0 \\
\hline If & $\mathrm{RN}=0.3$ & and & $\mathrm{L}=0.6$ & then & 1 & else & 0 \\
\hline If & $\mathrm{RN}=0.4$ & and & $\mathrm{L}=0.8$ & then & 1 & else & 0 \\
\hline If & $\mathrm{RN}=0.5$ & and & $\mathrm{L}=1$ & then & 1 & else & 0 \\
\hline If & $\mathrm{RN}=0.6$ & and & $\mathrm{LM}=0.2$ & then & 1 & else & 0 \\
\hline If & $\mathrm{RN}=0.7$ & and & $\mathrm{LM}=0.4$ & then & 1 & else & 0 \\
\hline If & $\mathrm{RN}=0.8$ & and & $\mathrm{LM}=0.6$ & then & 1 & else & 0 \\
\hline If & $\mathrm{RN}=0.9$ & and & $\mathrm{LM}=0.8$ & then & 1 & else & 0 \\
\hline If & $\mathrm{RN}=1$ & and & $\mathrm{LM}=1$ & then & 1 & else & 0 \\
\hline If & $\mathrm{RT}=0.1$ & and & $\mathrm{MH}=0.34$ & then & 1 & else & 0 \\
\hline If & $\mathrm{RT}=0.2$ & and & $\mathrm{MH}=0.67$ & then & 1 & else & 0 \\
\hline If & $\mathrm{RT}=0.3$ & and & $\mathrm{MH}=1$ & then & 1 & else & 0 \\
\hline If & $\mathrm{RT}=0.4$ & and & $\mathrm{H}=0.34$ & then & 1 & else & 0 \\
\hline If & $\mathrm{RT}=0.5$ & and & $\mathrm{H}=0.67$ & then & 1 & else & 0 \\
\hline If & $\mathrm{RT}=0.6$ & and & $\mathrm{H}=1$ & then & 1 & else & 0 \\
\hline If & $\mathrm{RT}=0.7$ & and & $\mathrm{EH}=0.34$ & then & 1 & else & 0 \\
\hline If & $\mathrm{RT}=0.8$ & and & $\mathrm{EH}=0.67$ & then & 1 & else & 0 \\
\hline If & $\mathrm{RT}=0.9$ & and & $\mathrm{EH}=0.99$ & then & 1 & else & 0 \\
\hline If & $\mathrm{RT}=1$ & and & $\mathrm{EH}=1$ & then & 1 & else & 0 \\
\hline
\end{tabular}

aggregating the output of the rules we utilize the Root Sum Square (RSS) method. In our case, as there are two predicates in the output membership function, the aggregation output of all the rules for each predicate is determined by:

$$
\text { 'Proceed' }=\sqrt{\Sigma(P)^{2}}
$$

'Don't Proceed' $=\sqrt{\Sigma(D P)_{2}}$

The determined values for each predicate from the aggregation process are then plotted on the output membership function, to ascertain the range of the output. The scalar output of the fuzzy inference system is then obtained by defuzzifying the range in which the output exists to obtain a crisp value by utilizing the centre of gravity or centroid method. The obtained crisp value when plotted on the output fuzzy set represents the recommended risk-based decision from the fuzzy inference model. If the risk assessing agent has to decide on an interaction when there is more than one risk assessed agent, then the fuzzy inference system repeats the above steps and determines the scalar output on the output membership function in forming an interaction with each risk assessed agent. In the next section we will explain the proposed methodology of risk-based decision making by an example.

\section{DETERMINING THE RECOMMENDED FUZZY RISK- BASED DECISION OUTPUT IN THE INTERACTION}

The main purposes of using a fuzzy inference system to assist the risk assessing agent in determining an interaction are:

- In contrast to the other approaches in the literature [1114], the fuzzy inference system allows the risk assessing agent to consider the intermediate states from the spectrum of the risk propensity levels, as its risk taking attitude in the interaction.

- It considers each level or predicate of the determined perceived risk in forming an interaction with a risk assessed agent, and ascertains the effect of its risk propensity on it, to determine the recommended risk-based decision in undertaking an interaction with that agent.

To explain the effectiveness of the fuzzy inference system while assisting the risk assessing agent in decision making, let us consider that a risk assessing agent ' $A$ ' has to choose a logistics company to fulfill its desired outcomes from a possible set of 3 companies, agent ' $\mathrm{B}$ ', agent ' $\mathrm{D}$ ' and agent ' $\mathrm{E}$ '. The risk assessing agent ' $\mathrm{A}$ ', in order to make a risk-based decision, analyses the level of perceived risk in interacting with each of the risk assessed agents by using the methodology explained in our previous work [8,9] and it determines the linguistic level of perceived risk in interacting with them as:

- Linguistic perceived risk in interacting with agent ' $\mathrm{B}$ ': $\mathrm{MH}=1, \mathrm{H}=0.1818, \mathrm{EH}=0.0909$.

- Linguistic perceived risk in interacting with agent ' $D$ ': $\mathrm{MH}=1, \mathrm{H}=0.9, \mathrm{EH}=0.5$.

- $\quad$ Linguistic perceived risk in interacting with agent ' $E$ ': $\mathrm{EL}=1, \mathrm{~L}=1, \mathrm{LM}=0.1$.

Let us consider that the risk assessing agent chooses a value of 3.6 as its 'Risk Propensity' in the interaction. While decision making, in order for the risk assessing agent to avoid being biased towards a particular agent and to assess all the risk assessed agents of the interaction in a uniform way, we propose that its risk propensity remains constant throughout the decision making process. By utilizing the proposed methodology, the range of output on the fuzzy inference in forming an interaction with each risk assessed agent is as shown in Figures 4-6.

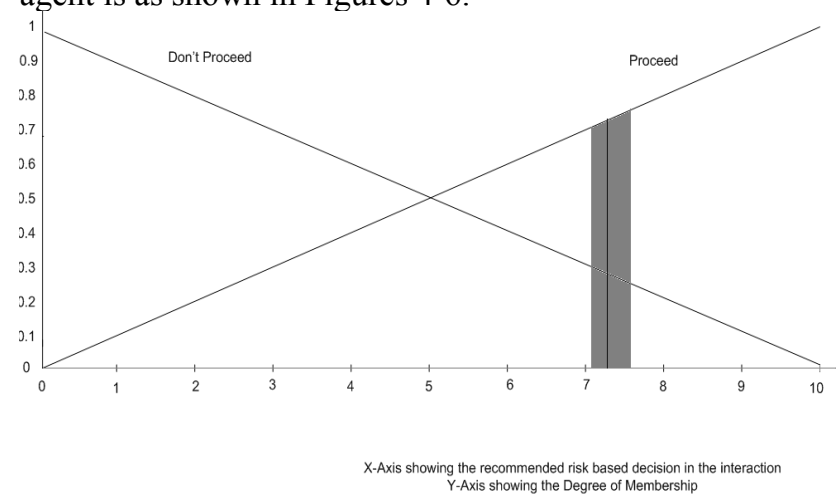

Figure 4: Range of the output fuzzy set in interacting with agent 'B'

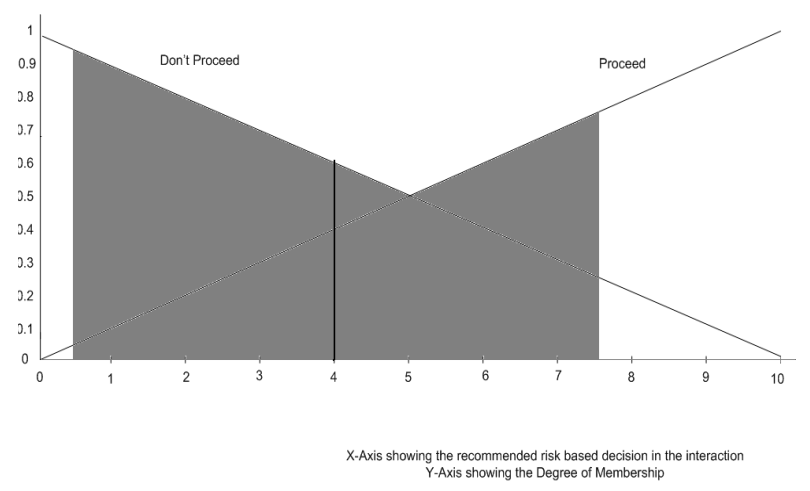

Figure 5: Range of the output fuzzy set in interacting with agent ' $D$ ' 


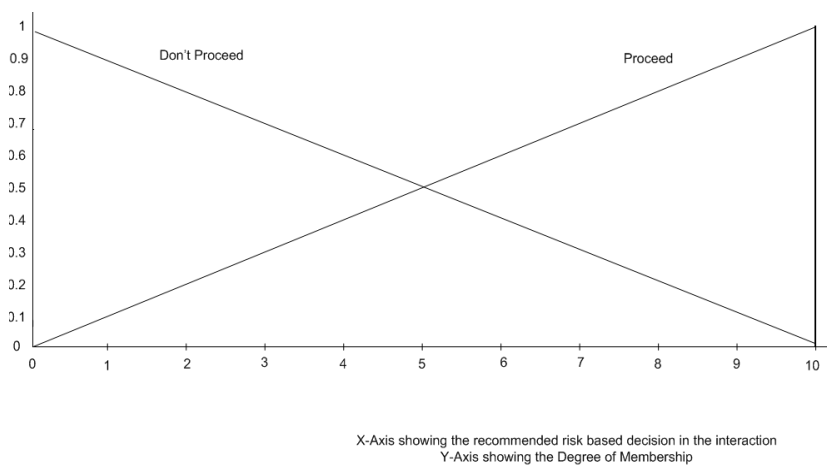

Figure 6: Range of the output fuzzy set in interacting with agent ' $E$ '

From the above figures it can be concluded that:

- The strength to which the rules correspond to the fuzzy set 'Proceed' are $73.69 \%, 40 \%$ and $100 \%$ while interacting with agent 'B', 'D' and ' $E$ ' respectively.

- Of the set of risk assessed agents, it can be concluded that the fuzzy inference system recommends agent ' $E$ ' to the risk assessing agent ' $\mathrm{A}$ ', as the strength by which the rules fires and produce an output leans towards the output fuzzy set 'Proceed' more while interacting with the other agents.

- In the example considered, it can be seen that even though the same rules fire in the case of agents ' $\mathrm{B}$ ' and ' $\mathrm{D}$ ', the strength to which each rule quantifies to its output set varies. This is due to the difference in the possibility of occurrence of the predicates of perceived risk in interacting with both the agents, which varies the output of the fuzzy inference system accordingly.

- While aggregating the fuzzy rules in interacting with agent ' $D$ ', it can be seen that if fuzzy additive method had been used, then the output fuzzy sets would have a DOM of 1.0 each. This would give the resultant answer from the fuzzy inference system as 0.5 'Proceed' and 0.5 'Don't Proceed'. Hence, those aggregation techniques were not used in this inference model and the RSS aggregation approach was used, which determines the accurate strength of the output fuzzy set by considering all the firing rules.

By utilizing the fuzzy recommended risk-based decision model, the risk assessing agent can make an informed decision about choosing an agent to interact with from a set of risk assessed agent, according to its risk propensity and the level and magnitude of the perceived risk in interacting with them. Further if the level of perceived risk in interacting with an agent is beyond its acceptable risk propensity, then the proposed approach will give the exact levels of variance. Based on that the risk assessing agent can determine the intensity by which it has to carry out the process of risk management. After the process of risk management the proposed approach can be utilized again to determine if the level of perceived risk has been reduced to be within acceptable limits according to its risk attitude.

\section{CONCLUSION}

In this paper we proposed a fuzzy inference system which assists the risk assessing agent to make an informed decision about an interaction with an interacting agent, based on the perceived risk in interacting with it. This is in contrast to the approaches in the literature which propose approaches for decision-making in the interaction just based on the level of trust. The inference system gives its recommendation by considering the impact of the risk attitude of the risk assessing agent on the determined levels of perceived risk according to its magnitude, in interacting with that interacting agent. Furthermore, the output which the fuzzy inference model gives is not just limited to an output predicate, like either 'Proceed' or 'Don't Proceed' but it also gives the strength to which each of the output fuzzy set quantifies. This is particularly useful for the risk assessing agent when it has to make an informed decision about choosing an agent with which to interact, from a set of interacting agents.

\section{REFERENCES}

[1] Elizabeth Chang, Tharam Dillon, and F. K. Hussain, Trust and Reputation for ServiceOriented Environments. West Sussex, England: John Wiley \& Sons, Ltd, 2006.

[2] Xiaoqing Zheng, Zhaohui Wu, Huajun Chen, and Y. Mao, "A Scalable Probabilistic Approach to Trust Evaluation," in Fourth International Conference on Trust Management (iTrust'06), Pisa, Italy, 2006, pp. 423-438.

[3] Audun Jøsang, Claudia Keser, and T. Dimitrakos, "Can We Manage Trust?," in Third International Conference on Trust Management (iTrust 2005), Rocquencourt, France, 2005, pp. 93-107.

[4] Lewis Hassell, "Affect and Trust," in Third International Conference on Trust Management (iTrust'05), France, 2005, pp. 131-145.

[5] Siani Pearson, Marco Casassa Mont, and S. Crane, "Persistent and Dynamic Trust: Analysis and the Related Impact of Trusted Platforms," in Third International Conference on Trust Management (iTrust'05), France, 2005, pp. 355-363.

[6] Omar Khadeer Hussain, Elizabeth Chang, Farookh Khadeer Hussain, and Tharam S. Dillon, "A methodology to quantify failure for risk-based decision support system in digital business ecosystems," Data \& Knowledge Engineering, vol. 63, pp. 597-621, December 2007.

[7] Omar K. Hussain, Elizabeth Chang, Farookh K. Hussain, and Tharam S. Dillon, "Towards Quantifying the Possible Risk in e-commerce Interactions for RDSS," in Proceedings of the IEEE International Conference on e-Business Engineering (ICEBE'07), IEEE, Ed. Hong Kong, China: IEEE, 2007, pp. 89-96.

[8] Omar K. Hussain, Elizabeth Chang, Tharam S. Dillon, and Farookh K. Hussain, "Determining the Level of Perceived Risk in e-business Web 2.0 Interactions," in IEEE International Conference on e-Business Engineering (ICEBE'08), IEEE, Ed. Xian, China: IEEE, 2008, pp. 89-96.

[9] Omar K. Hussain, Elizabeth Chang, Tharam S. Dillon, and Farookh K. Hussain, "Ascertaining the Semantic and Linguistic Level of Perceived Risk in e-Business Interactions," in IEEE International Conference on e-Business Engineering (ICEBE'08), IEEE, Ed. Xian, China: IEEE, 2008, pp. 723-727.

[10] Sim B. Sitkin and L. R. Weingart, "Determinants of Risky Decision-Making Behavior: A Test of the Mediating Role of Risk Perceptions and Propensity," Academy of Management Journal, vol. 38, pp. 1573-1592, December 1995.

[11] Ching Lin and V. Varadharajan, "Trust Based Risk Management for Distributed System Security - A New Approach," in The First International Conference on Availability, Reliability and Security (ARES'06) Vienna: IEEE Computer Society, 2006, pp. 6-13.

[12] Dariusz Wawrzyniak, "Information Security Risk Assessment Model for Risk Management," in Third International Conference on Trust, Privacy and Security in Digital Business (TrustBus'06) Krakow, Poland: LNCS Vol 4083, 2006, pp. 21-30.

[13] Christian Schläger and T. Nowey, "Towards a Risk Management Perspective on AAIs," in Third International Conference on Trust and Privacy in Digital Business (TrustBus'06). vol. Vol 4083 Krakow, Poland: LNCS 2006, pp. 41-50.

[14] Juan Xu, Zhixue Liu, and Y. Li, "Integrating Processes of Logistics Outsourcing Risk Management in e-Business," in IEEE/WIC/ACM International Joint Conference on Web Intelligence and Intelligent Agent Technology Hong Kong, 2006, pp. 544-547 